\title{
Projection of diffraction patterns for use in cold-neutral-atom trapping
}

\author{
Katharina Gillen-Christandl* and Glen D. Gillen \\ Physics Department, California Polytechnic State University, San Luis Obispo, California 93407, USA
}

(Received 13 September 2010; published 29 December 2010)

\begin{abstract}
Scalar diffraction theory is combined with beam-propagation techniques to investigate the projection of near-field diffraction patterns to spatial locations away from the aperture for use in optically trapping cold neutral alkali-metal atoms. Calculations show that intensity distributions with localized bright and dark spots usually found within a millimeter of the diffracting aperture can be projected to a region free from optical components such as a cloud of cold atoms within a vacuum chamber. Calculations also predict that the critical properties of the optical dipole atom traps are not only maintained for the projected intensity patterns but also can be manipulated and improved by adjustment of the optical components outside the vacuum chamber.
\end{abstract}

DOI: 10.1103/PhysRevA.82.063420

PACS number(s): 37.10.De, 42.25.Fx, 42.25.Bs

\section{INTRODUCTION}

When an atom is exposed to the oscillating electric field of laser light, the light field can exert an optical dipole force on the atom. The first experimental observation of the optical dipole force was achieved in 1978 by Bjorkholm et al. [1]. Since that time, several fields of study have developed which exploit the optical dipole force on cold atoms and molecules. One application of the optical dipole force is to confine and trap cold neutral atoms within spatially varying light field distributions [2]. If the frequency of the laser light is less than the resonant frequency of the atom, the dipole potential energy is a minimum for localized high-intensity fields, and a red-detuned atomic trap (RDT) is created. Conversely, if the frequency of the laser light is greater than the resonant frequency of the atom, the dipole potential energy is a minimum for localized low-intensity fields, and a blue-detuned atomic trap (BDT) is created [3]. The difference between the various pursuits within the field of trapping cold neutral atoms is determined by the optical methods employed to create laser light fields with localized maxima or minima. Various methods to create localized high- or low-intensity fields have included the focusing of a single laser beam [4] to create a single trap at the focal spot; using the periodic interference pattern of counterpropagating beams to create one-dimensional [5], two-dimensional [6], and three-dimensional [7] arrays of optical traps; using axicons [8] or Laguerre-Gaussian beams [9] to create localized dark regions; using evanescent-wave traps [10]; using the interference of different evanescent waves outside of a waveguide from different propagation modes within the waveguide to create one- and two-dimensional arrays of optical traps [11].

Another method of recent interest for creating light fields with localized maxima and minima for trapping atoms is to use the diffracted light in and around spatially limiting apertures [12-15]. Klimov and Letokhov first theoretically investigated trapping atoms in light distributions, formed by interference of incident and scattered light, located before and within diffracting apertures with dimensions smaller than the wavelength of light [12]. In our earlier work, we investigated

\footnotetext{
*kgillen@calpoly.edu
}

diffracted light distributions beyond a circular aperture with dimensions larger than the wavelength of light using Hertz vector diffraction theory to create either RDTs or BDTs, and we investigated their associated trapping properties [13]. Similar calculations were performed by Bandi et al. using Rayleigh-Sommerfeld diffraction theory [14]. Chen and Yin further theoretically improved the properties of trap sites created by diffracted laser light by use of a binary phase plate in addition to the diffracting aperture [15].

Using diffracted laser light for the trapping of cold atoms allows for an experimental setup with (1) a fairly simple optical setup comprising an unfocused, or loosely focused, laser beam and a diffracting aperture or mask and (2) the ability to use the same optical setup for both RDTs and BDTs by changing only the detuning of the laser. However, there are two issues which make experimental implementation of using diffracted laser light to create red-detuned and blue-detuned optical dipole traps challenging. First, the location of the diffraction patterns is very close to the diffracting aperture. Experimentally, this would mean that the diffracting aperture would have to be placed within the vacuum chamber so that cold atoms could be loaded into the diffraction pattern locations. The diffracting aperture would need to be included with the design and construction of the vacuum chamber and the optical system for the chosen atom cooling technique. For example, one commonly used technique is a magneto-optical trap (MOT). Experimentally, adjustment or replacement of the diffracting aperture or mask would require a significantly time-consuming process of venting and disassembly of the vacuum chamber, adjustment and/or replacement, cleaning the vacuum chamber, baking the vacuum chamber, and waiting for it to reach the ultra-high vacuum (UHV) level required for atomic trapping. The second main experimental challenge for implementation of diffraction-based atom trapping is the fact that the diffraction pattern just beyond the aperture (and consequently the trap size, shape, trap frequencies, etc.) is a fixed function of the chosen aperture. Adjustment of the trap properties would require the replacement of the diffracting aperture or mask and all of the complexities involved in doing so.

In this article, we present an optical method, a mathematical model, and computational results for projecting the diffraction 
pattern located just beyond a diffracting aperture to another location away from optical components, that is, inside a MOT cloud of cold atoms inside a UHV chamber. This method eliminates the two experimental challenges of using diffracted light to trap atoms. First, by projecting the pattern away from the diffracting aperture, the need to place the aperture inside the vacuum chamber is eliminated, and all optical components would be located outside the chamber, where they can be easily aligned, adjusted, and/or replaced. Second, it is demonstrated here that adjustment of the aperture-to-lens distance allows for control and adjustment of the size and depth of the optical traps for a fixed diffracting aperture. If a different aperture is desired, exchanging it is a quick and straightforward process, as it is located outside the vacuum chamber. The method presented here is only for the projection of a single aperture yielding a single RDT or BDT site within the MOT cloud. However, the method is extendable for the projection of a more complex diffracting mask of a one- or two-dimensional array of apertures yielding a projected array of trap sites.

\section{DIFFRACTION AND LIGHT-PROPAGATION THEORY}

\section{A. Choosing a model}

In general, the difference between various established diffraction models is which mathematical approximations can be assumed in order to decrease both the mathematical complexity and computational times. The location of the point of interest with respect to the plane or surface area of the diffracting aperture to be integrated determines which mathematical approximations are valid. The closer the point of interest is to the diffracting aperture, the fewer the number of valid mathematical approximations, and hence the more complicated the diffraction model. One model which employs very few approximations (i.e., an infinitely thin and perfectly conducting aperture) and whose region of validity includes all points within the aperture plane and beyond is Hertz vector diffraction theory (HVDT) [16,17]. Therefore HVDT is the diffraction model used in this work to calculate the diffraction pattern in the region of interest just beyond the circular aperture. The results

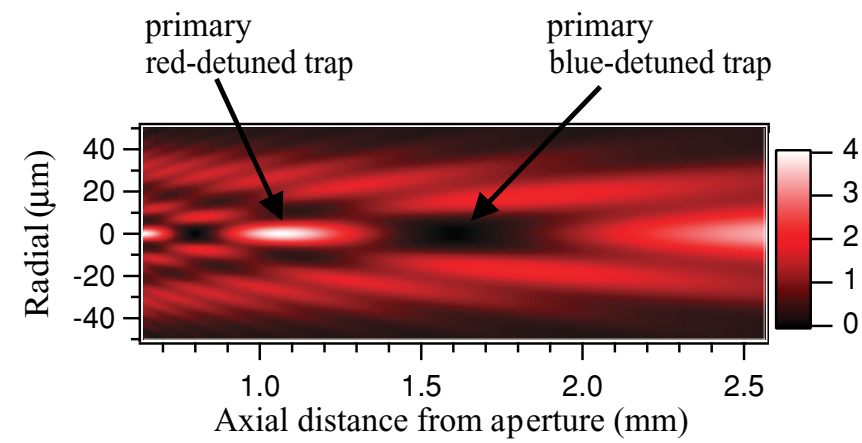

FIG. 1. (Color online) Intensity distribution pattern just beyond a metallic circular aperture with a radius of $50 \mu \mathrm{m}$ and a laser wavelength of $780 \mathrm{~nm}$, normalized to the intensity incident upon the aperture. The locations of the localized bright region for the primary red-detuned trap site and the localized dark region for the primary blue-detuned trap site are noted. are depicted in Fig. 1. The parameters used to calculate Fig. 1 are an aperture radius of $50 \mu \mathrm{m}$ and a laser wavelength of $780 \mathrm{~nm}$. It should be noted here that for a circular aperture of radius $a$ and a laser wavelength of $\lambda$, there will be $a / \lambda$ on-axis bright spots and $a / \lambda-1$ on-axis dark spots [18]. For the purposes of this investigation, the dark spot farthest from the aperture is referred to as the primary BDT, and the second to last bright spot farthest from the aperture is referred to as the primary RDT, as noted in Fig. 1. Of all of the on-axis maxima and minima, these two regions are chosen for investigation because they are the largest of each, which makes them easier to identify, load with cold atoms, and probe the atoms trapped within them in initial experiments. Both the primary BDT and the primary RDT lie within the region of validity of Fresnel approximations [18], or

$$
z_{1}^{3} \gg \frac{\pi}{4 \lambda}\left[\left(x_{1}-x_{0}\right)^{2}+\left(y_{1}-y_{0}\right)^{2}\right]^{2}
$$

where $x_{1}, y_{1}$, and $z_{1}$ are the coordinates of the point of interest and $x_{0}$ and $y_{0}$ are the locations of an integration point in the aperture plane. Therefore the diffraction model used to calculate the projected diffraction patterns is the Fresnel integral diffraction model. For a complete discussion of the Fresnel integral model, see Refs. [18,19].

\section{B. Diffraction and beam-propagation model}

Figure 2 illustrates the theoretical setup for this investigation, where a single lens of focal length $f$ is placed a distance $L$ from a circular aperture of radius $a$ to project the diffraction pattern from just behind the metallic aperture into a MOT cloud of cold atoms located within an optics-free region inside the UHV chamber.

For the theoretical setup depicted in Fig. 2, the Fresnel approximation for the diffracted fields at a point in the nearfield diffraction region [Eqs. (4)-(17) of Ref. [19]] can be

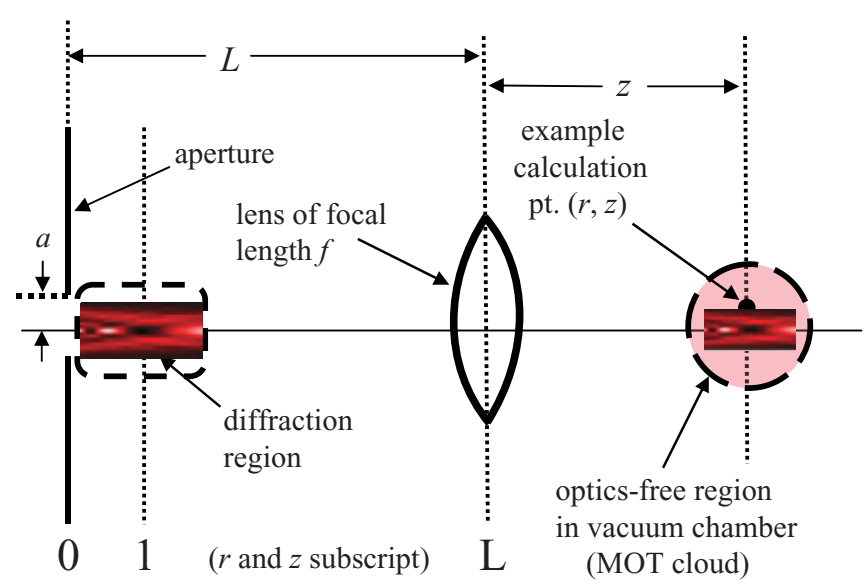

FIG. 2. (Color online) Theoretical setup for projecting near-field diffraction patterns to an optics-free location within a MOT cloud of cold atoms. The key parameters are the aperture radius, $a$, the aperture-to-lens distance, $L$, the focal length of the lens, $f$, and the axial distance between the lens and the point of interest, $z$. Plane 0 is the axial location of the aperture, and plane 1 is an axial plane within the diffraction region. 
expressed as

$$
\begin{aligned}
E_{1}\left(x_{1}, y_{1}, z_{1}\right)= & \frac{k e^{i k z_{1}}}{i 2 \pi z_{1}} \exp \left[i \frac{k}{2 z_{1}}\left(x_{1}^{2}+y_{1}^{2}\right)\right] \\
& \times \iint_{-\infty}^{\infty} E_{z_{0}} \exp \left[i \frac{k}{2 z_{1}}\left(x_{0}^{2}+y_{0}^{2}\right)\right] \\
& \times \exp \left[-i \frac{k}{z_{1}}\left(x_{1} x_{0}+y_{1} y_{0}\right)\right] d x_{0} d y_{0},
\end{aligned}
$$

where $k$ is the wave number, or $2 \pi / \lambda,\left(x_{1}, y_{1}, z_{1}\right)$ is the location of the point of interest in the diffraction region, $\left(x_{0}, y_{0}, z_{0}\right)$ is an integration point in the aperture, and $E_{z_{0}}$ is the distribution of the incident electric field within the open area of the aperture. Here $E_{z 0}$ is assumed to be a constant to represent an incident plane wave (i.e., a loosely focused beam with a width $\gg a$ ). For the diffraction of light by a circular aperture, the net light fields within the aperture plane do not have cylindrical symmetry as a result of scattering effects of the aperture rim due to the vector field polarization direction [16,17]. However, for points with axial distances greater than the aperture radius, the diffracted light fields do exhibit cylindrical symmetry [17]. Converting Eq. (2) to cylindrical coordinates and assuming cylindrical symmetry, the field beyond the aperture in the Fresnel diffraction region becomes

$$
\begin{aligned}
E_{1}\left(r_{1}, z_{1}\right)= & \frac{k e^{i k z_{1}}}{i z_{1}} \exp \left[i \frac{k r_{1}^{2}}{2 z_{1}}\right] \\
& \times \int_{0}^{a} E_{z_{0}} J_{0}\left(\frac{k r_{0} r_{1}}{z_{1}}\right) \exp \left[i \frac{k r_{0}^{2}}{2 z_{1}}\right] r_{0} d r_{0},
\end{aligned}
$$

where $J_{0}$ is a Bessel function of the first kind of order zero.

The effect of the lens on the electric field can be approximated using a phase transformation of the light fields incident upon the lens [19]. For the lenses and optical setups used in this investigation, the paraxial thin lens phase transformation can be used. The electric field leaving the lens, $E_{L}$, in the cylindrical coordinate system of the lens, $\left(r_{L}, z_{L}\right)$, the origin of which is at the center of the lens, is related to the field incident upon the lens, $E_{1}$, in the diffraction plane coordinate system, $\left(r_{1}, z_{1}\right)$, by

$$
E_{L}\left(r_{L}, z_{L}=0\right)=E_{1}\left(r_{1}, z_{1}=L\right) t\left(r_{L}\right),
$$

where $L$ is the axial distance between the diffracting aperture and the lens and $t\left(r_{L}\right)$ is the thin lens phase transformation as a function of radial distance from the optical axis. The thin lens phase transformation for a lens of focal length $f$ is given by Eqs. (5)-(10) of Ref. [19], or

$$
t\left(r_{L}\right)=\exp \left[-i \frac{k r_{L}^{2}}{2 f}\right]
$$

using cylindrical coordinates with cylindrical symmetry. Substituting Eqs. (3) and (5) into Eq. (4), the field at a single point in space immediately after the lens becomes

$$
\begin{aligned}
E_{L}\left(r_{L}, 0\right)= & \frac{k e^{i k L}}{i L} \exp \left[i \frac{k r_{L}^{2}}{2 L}\right] \int_{0}^{a} E_{z_{0}} J_{0}\left(\frac{k r_{0} r_{L}}{L}\right) \\
& \times \exp \left[i \frac{k r_{0}^{2}}{2 L}\right] \exp \left[-i \frac{k r_{L}^{2}}{2 f}\right] r_{0} d r_{0},
\end{aligned}
$$

where $r_{L}$ is the radial location of a point within the plane of the center of the lens. To obtain the projected electric field at the point of interest in the optics-free region of the MOT cloud of cold atoms, it is necessary to integrate the electric field just after the lens over the open area of the lens and propagate the fields to the point of interest. Using the Fresnel diffraction integral, the field at the point of interest, $(r, z)$, where the coordinate system has the same origin as the lens coordinate system, becomes a function of the field distribution just after the lens:

$$
\begin{aligned}
E(r, z)= & \frac{k e^{i k z}}{i z} \exp \left[i \frac{k r^{2}}{2 z}\right] \int_{0}^{R} E_{L}\left(r_{L}, L\right) \\
& \times J_{0}\left(\frac{k r_{L} r}{z}\right) \exp \left[i \frac{k r_{L}^{2}}{2 z}\right] r_{L} d r_{L},
\end{aligned}
$$

where the integration is over the open area of the lens and $R$ is the radius of the lens. Substitution of Eq. (6) into Eq. (7) yields

$$
\begin{aligned}
E(r, z)= & -\frac{k^{2} e^{i k(z+L)}}{L z} \exp \left(i \frac{k r^{2}}{2 z}\right) \\
& \times \int_{0}^{R}\left[\int_{0}^{a} E_{z_{0}} J_{0}\left(\frac{k r_{0} r_{L}}{L}\right) \exp \left(i \frac{k r_{0}^{2}}{2 L}\right) r_{0} d r_{0}\right] \\
& \times J_{0}\left(\frac{k r_{L} r}{z}\right) \exp \left[i \frac{k}{2}\left(\frac{1}{L}+\frac{1}{z}-\frac{1}{f}\right) r_{L}^{2}\right] r_{L} d r_{L} .
\end{aligned}
$$

If the lens size is significantly larger than the diffraction pattern incident upon the lens, then the limit of the $\mathrm{d} r_{L}$ integral can be assumed to be infinity. The integral of the lens plane can then be evaluated explicitly, and Eq. (8) can be simplified to

$$
\begin{aligned}
E(r, z)= & \frac{d k e^{i k(z+L)}}{i L z} \exp \left[i \frac{k}{2 z}\left(1-\frac{d}{z}\right) r^{2}\right] \\
& \times \int_{0}^{a} E_{z_{0}} J_{0}\left(\frac{k r_{0} r d}{L z}\right) \\
& \times \exp \left[i \frac{k}{2 L}\left(1-\frac{d}{L}\right) r_{0}^{2}\right] r_{0} d r_{0},
\end{aligned}
$$

where the parameter $d$ is defined to be

$$
d=\left[\frac{1}{L}+\frac{1}{z}-\frac{1}{f}\right]^{-1} .
$$

Equation (9) is the general integral result of this article, yielding the electric field at the point of interest in the optics-free region within the MOT cloud. It should be noted here that the axial distance from the lens which forces the value of $d$ to approach infinity is the axial location of the image of the aperture plane according to geometrical optics.

\section{On-axis fields}

While the general integral result of Eq. (9) is complete and yields the scalar light field for any point within the desired projection region, it can be cumbersome to quickly determine where the projected light distributions can be found. The 
mathematics of the general integral result of Eq. (9) simplify for on-axis locations, $r=0$, and the integral can be directly evaluated, yielding the more convenient analytical form

$$
\begin{aligned}
E(0, z)= & -\frac{E_{z_{0}} d}{z\left(1-\frac{d}{L}\right)} e^{i k(z+L)} \\
& \times\left\{\exp \left[i \frac{k a^{2}}{2 L}\left(1-\frac{d}{L}\right)\right]-1\right\},
\end{aligned}
$$

which is dependent upon the wave number of the light, $k$, the aperture radius, $a$, the distance between the aperture and the lens, $L$, and the focal length of the lens, $f$. The on-axis projected scalar field of Eq. (11) only exhibits localized maxima and minima around the region of the location of the imaged near-field pattern, according to geometrical optics. Equation (11) can be evaluated to obtain the locations of the on-axis maxima and minima. The exact locations of on-axis maxima and minima occur when

$$
z=f \frac{1-n L \frac{\lambda}{a^{2}}}{1-n(L-f) \frac{\lambda}{a^{2}}},
$$

where $n$ is a nonzero integer. On-axis maxima correspond to when $n$ is an odd integer, and on-axis minima correspond to when $n$ is an even integer. On-axis maxima represent locations of possible projected RDTs, and on-axis minima represent locations of possible projected BDTs. Equation (12) can also be written in the more familiar style of a thin lens equation in geometrical optics, or

$$
\frac{1}{z}+\frac{1}{L-\frac{a^{2}}{n \lambda}}=\frac{1}{f} .
$$

For the two on-axis locations of interest for this investigation, a value of $n=2$ corresponds to the projection of the primary BDT, and a value of $n=3$ corresponds to the projection of the primary RDT.

\section{ATOM-TRAPPING POTENTIAL ENERGY}

When an atom is exposed to an electromagnetic field, the interaction of the light and the induced dipole moment of the atom results in a dipole potential energy and its associated dipole force. For detunings that are large compared to the excited state hyperfine splitting but small compared to the fine structure splitting, and for linearly polarized light, the positiondependent potential energy of this interaction for an atom in a hyperfine ground state $F$ can be expressed as [20]

$$
U(\mathbf{r})=\beta \frac{\hbar \Gamma}{8} \frac{\Gamma}{\Delta} \frac{|E(\mathbf{r})|^{2}}{\left|E_{s}\right|^{2}},
$$

where $\mathbf{r}$ is the location of the point of interest, $\beta$ is the linestrength factor of the atomic transition $[2,21], \Gamma$ is the spontaneous decay rate, $E_{s}$ is the saturation field of the transition, and $\Delta$ is the laser detuning from the transition from $F$ to the center of the excited state hyperfine manifold. The detuning, $\Delta$, is the difference between the angular frequency of the light field, $\omega$, and the angular frequency of a photon in resonance with the atomic transition, $\omega_{o}$, or

$$
\Delta=\omega-\omega_{o} .
$$

The particular cold neutral atoms used for this study are ${ }^{85} \mathrm{Rb}$ atoms, and the specific transition of interest is the $D 2$ transition $\left({ }^{2} S_{1 / 2} \rightarrow{ }^{2} P_{3 / 2}\right)$, which has a line-strength factor of $\beta=2 / 3$. Including the line-strength factor and the cylindrical symmetry of the diffracted light fields, the atom-trapping potential energy becomes

$$
U(r, z)=\frac{2}{3} \frac{\hbar \Gamma}{8} \frac{\Gamma}{\Delta} \frac{|E(r, z)|^{2}}{\left|E_{s}\right|^{2}} .
$$

For cold ${ }^{85} \mathrm{Rb}$ atoms and $780 \mathrm{~nm}$ laser light, the saturation electric field for this transition is $E_{s}=111.5 \mathrm{~V} / \mathrm{m}$ [22] (or an intensity of $16.5 \mathrm{~W} / \mathrm{m}^{2}$ ), and the spontaneous decay rate is $\Gamma=2 \pi \times 6 \mathrm{MHz}[22]$.

The magnitude of the optical potential energy of the atom is directly proportional to the intensity of the laser field. The sign of the potential energy is determined by the sign of the detuning. If $\omega<\omega_{o}$, the energy of a photon in the laser field is less than the energy of the atomic transition, the potential energy is negative and becomes more negative for higher intensity locations, and the atom is drawn toward regions of higher intensity light and RDTs are created. If the detuning is positive, or $\omega>\omega_{o}$, the atoms are drawn toward regions of lower intensity light and, consequently, BDTs are created.

\section{PROJECTION CALCULATIONS}

\section{A. Intensity calculations}

The method introduced in this article allows for the remote control and manipulation of the optical dipole traps within the vacuum chamber by placing the diffracting aperture and projection lens outside the vacuum chamber. Without breaking vacuum, the placement, radial and axial sizes of the trap sites as well as the trap depths can be adjusted and controlled for a given laser frequency by changing any one of three optical parameters: the aperture radius, $a$, the focal length of the lens, $f$, and the aperture-to-lens distance, $L$.

Figure 3 illustrates some of the control over the projected diffraction pattern by adjustment of only the parameter $L$. All the image plots for Fig. 3 are for a wavelength of $780 \mathrm{~nm}$, an aperture radius of $25 \mu \mathrm{m}$, and a focal length of $40 \mathrm{~mm}$. The numerical intensity scale of each part of Fig. 3 is normalized to the intensity of the laser light incident upon the diffracting aperture. Figure 3(a) is a calculation of the fields just beyond the aperture using HVDT. Figure 3(b) is an image plot of a projected diffraction pattern, using Eq. (9), and approximately equal in size to that of the original. To project a diffraction pattern equal to that of the original pattern in physical size and relative intensity, an aperture-to-lens distance of just over twice the focal length is chosen, or $L=80.4 \mathrm{~mm}$. This particular choice forces the size of the projected pattern to approximately equal that of the original and forces the distance of the projected BDT from the lens to equal exactly the distance of the original BDT to the lens. The relative intensity of the projected pattern is equal to that of the original pattern, where the intensity of the RDT is 4 times that of the incident laser intensity. Increasing the aperture-to-lens distance beyond $2 f$ has two effects upon the projected pattern: The entire projected pattern will be smaller than the original and, 
(a)

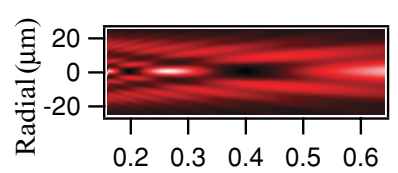

(b)

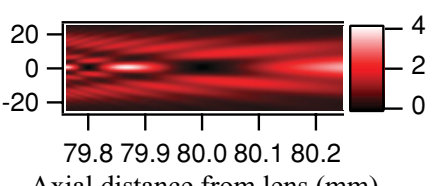

Axial distance from aperture (mm) Axial distance from lens (mm)

(c)

(d)

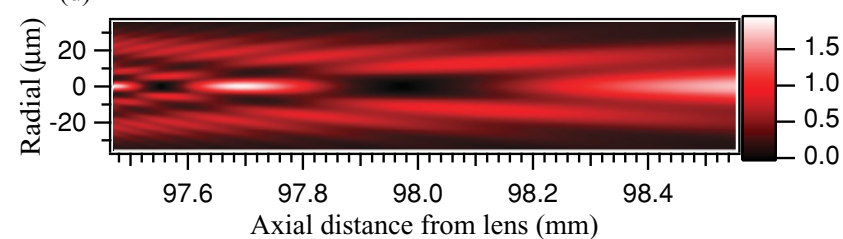

(e)

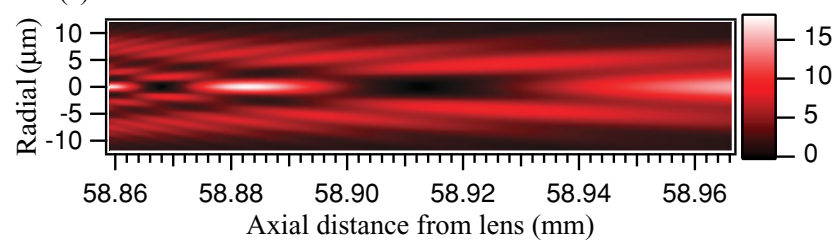

FIG. 3. (Color online) Diffraction patterns for an aperture with a radius of $25 \mu \mathrm{m}$, where (a) is the original pattern just beyond the diffracting aperture, (b) is a projection with the aperture-to-lens distance, $L$, chosen such that the projection is approximately the same size as the original, (c) is a projection with $L$ chosen such that the projection is smaller than the original, and (d) is a projection with $L$ chosen such that the projection is larger than the original. Parts (b)-(d) are all set to the same distance scaling as (a) for direct comparison of the size differences, and (e) is an expansion of (c). All numerical intensity scaling values are normalized to the intensity incident upon the aperture.

consequently, the relative intensity of the bright spots will be amplified with respect to the incident laser intensity, as illustrated in Figs. 3(c) and 3(e), where $L$ is set to be $125 \mathrm{~mm}$. Conversely, by adjusting the aperture-to-lens distance to values less than $2 f$, the projected pattern will be larger than the original and have a lower overall intensity, as illustrated in Fig. 3(d), where $L$ is set to be $68 \mathrm{~mm}$. Both Figs. 3(c) and 3(d) are intentionally set to the same radial scaling and axial scaling as Fig. 3(a) to better illustrate the effects of changing $L$ on the overall projected pattern size. Figure $3(\mathrm{e})$ is an expanded view of the smaller projection of the diffraction pattern [Fig. 3(c)]. Note here the differences in radial and axial sizes of the projected traps, as compared to the original trap sites of Fig. 3(a). In addition, the peak intensity of the projected RDT is now 17.9 times the incident laser intensity, whereas that of Fig. 3(d) is only 1.93 times the incident laser intensity.

With the diffracting aperture located outside the vacuum chamber, exchanging the aperture for one with a different radius is a straightforward process. Changing the size of the aperture radius affects the radial and axial scalings of the nearfield diffraction pattern differently; the radial dimensions of the pattern scale linearly with the aperture radius, whereas the axial scaling of the pattern is dependent upon the square of the aperture radius [18]. For circular apertures, where the radius (a)

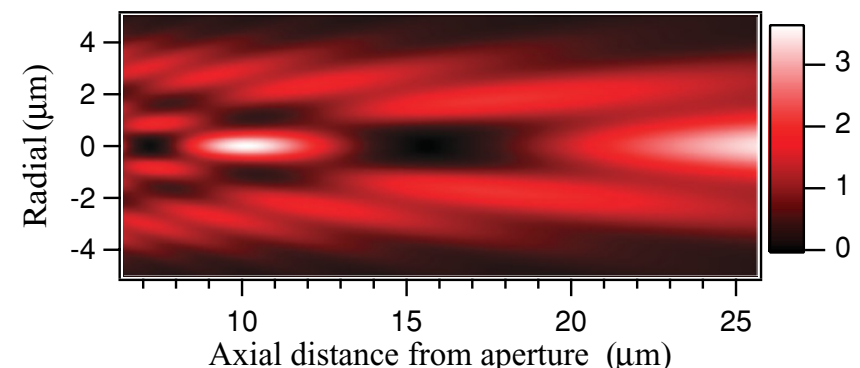

(b)

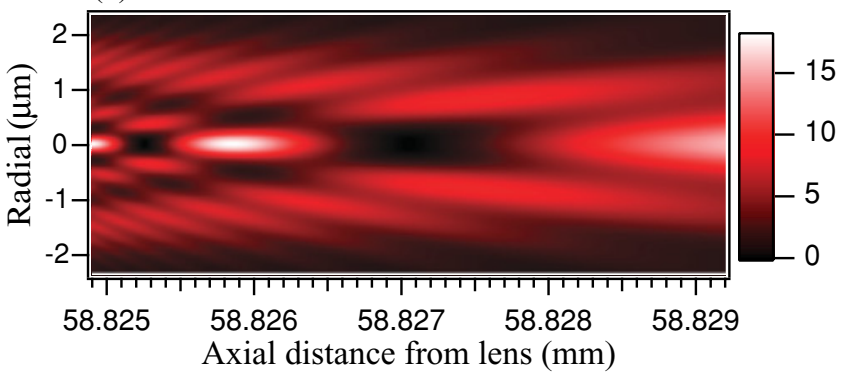

FIG. 4. (Color online) (a) Original and (b) projected intensity patterns for the creation of a nanotrap. The aperture radius is $5 \mu \mathrm{m}$, the focal length of the lens is $40 \mathrm{~mm}$, and the aperture-to-lens distance is set to $125 \mathrm{~mm}$. Both intensity scaling values are normalized to the intensity incident upon the aperture.

is much larger than the wavelength of the laser light, such as those of Fig. 3, where $a / \lambda=32$, the trap sites tend to be much longer in the axial dimension than in the radial dimension. Because of the different dependencies of the radial and axial scalings on the aperture radius, the aspect ratio of the radial size to the axial size increases as the size of the diffracting aperture decreases. It should be noted here that care must be taken when choosing smaller apertures to remain within the region of validity of the Fresnel approximations used in this model, where it is assumed that $a \gg \lambda$. With the assumptions used for this model and the locations of the primary RDT and primary BDT, a lower limit on the aperture size would be an aperture size of about $5 \mu \mathrm{m}$, where $a / \lambda=6.25$. Figure 4(a) is an image plot of the intensity pattern just beyond a $5 \mu \mathrm{m}$ radius circular aperture using HVDT. Sub-micron-sized traps, or nanotraps, can be created by using our projection technique. As $L$ becomes larger than $2 f$, the projected pattern becomes smaller than the original diffraction pattern. Figure $4(\mathrm{~b})$ is an illustration of the creation of a nanotrap. First, we start with Fig. 4(a), or the smallest original diffraction pattern within the region of validity of the projection model used here, and then we set $L>2 f$. For this particular illustration, $f$ is chosen to be a $40 \mathrm{~mm}$ lens, and $L$ is chosen to be $125 \mathrm{~mm}$, which yields RDT and BDT axial trap dimensions of $\approx 1 \mu \mathrm{m}$ and radial trap dimensions of $<1 \mu \mathrm{m}$.

\section{B. Trapping potential energy calculations}

Conversion of the intensity distributions of Figs. 3 and 4 to potential energy wells for trapping cold neutral atoms requires a choice of the laser detuning and an incident laser intensity. Additionally, for detunings not large compared to 
TABLE I. Properties of the primary RDT for various diffraction patterns. For all patterns, the lens focal length is $40 \mathrm{~mm}$, the detuning parameter is set to $=-10^{4} \Gamma$, and the trap depth is given per $100 \mathrm{~W} / \mathrm{m}^{2}$ of light intensity incident upon the aperture. For reference, a $17 \mathrm{~mW}$ laser focused to a spot diameter of $\sim 150 \mu \mathrm{m}$ gives an incident intensity of $\sim 100 \mathrm{~W} / \mathrm{m}^{2}$.

\begin{tabular}{|c|c|c|c|c|}
\hline Diffraction pattern & $\begin{array}{l}\text { Figure } \\
\text { number }\end{array}$ & $\begin{array}{c}\text { RDT trap depth } \\
\text { per } 100 \mathrm{~W} / \mathrm{cm}^{2} \\
(\mu \mathrm{K})\end{array}$ & $\begin{array}{l}\text { Radial width } \\
\qquad(\mu \mathrm{m})\end{array}$ & $\begin{array}{l}\text { Axial length } \\
\quad(\mu \mathrm{m})\end{array}$ \\
\hline \multicolumn{5}{|l|}{ Original } \\
\hline$a=25 \mu \mathrm{m}$ & 3(a) & 277 & 6.03 & 120 \\
\hline Projected & & & & \\
\hline $\begin{array}{l}a=25 \mu \mathrm{m}, L=80.4 \mathrm{~mm} \\
\text { Projected }\end{array}$ & 3(b) & 280 & 6.03 & 120 \\
\hline $\begin{array}{l}a=25 \mu \mathrm{m}, L=125 \mathrm{~mm} \\
\text { Projected }\end{array}$ & $3(\mathrm{c}), 3(\mathrm{e})$ & 1250 & 2.84 & 26.7 \\
\hline $\begin{array}{l}a=25 \mu \mathrm{m}, L=68 \mathrm{~mm} \\
\text { Original }\end{array}$ & $3(d)$ & 134 & 8.88 & 252 \\
\hline$a=5 \mu \mathrm{m}$ & 4(a) & 241 & 1.31 & 4.89 \\
\hline $\begin{array}{l}\text { Projected nanotrap } \\
a=5 \mu \mathrm{m}, L=125 \mathrm{~mm}\end{array}$ & 4(b) & 1260 & 0.59 & 1.08 \\
\hline
\end{tabular}

the hyperfine ground-state splitting, a choice of a hyperfine ground state of the atom is required. Using the same detuning as our previous work [13] $\left(=-10^{4} \Gamma\right.$ for RDT sites and $=10^{3} \Gamma$ from the $F=2$ hyperfine ground state for BDT sites), disturbances of the possible atom traps because of photon absorption, population of excited states, and the spontaneous force are negligible. Including the saturation field for the chosen transition, the trap potential energy [Eq. (16)] can be expressed as a function of the chosen detuning and normalized to the intensity incident upon the aperture, or

$$
\frac{U(r, z)}{I_{o}}=9.70 \times 10^{3} \frac{\Gamma}{-}\left(\frac{I(r, z)}{I_{o}}\right) \frac{\mu \mathrm{K}}{\mathrm{W} / \mathrm{cm}^{2}},
$$

where the term $I / I_{o}$ is the normalized intensity displayed in the numerical intensity scaling of Figs. 3 and 4.

\section{Properties of calculated traps}

Tables I and II give the trap depths and trap dimensions for all the diffraction patterns illustrated in Figs. 3 and 4. The depth of each trap site is taken to be the difference between the minimum of the trap potential well and the lowest potential barrier to getting out of a RDT or a BDT along the escape path. (For a complete discussion on escape paths from each type of trap, see our previous work [13].) The radial and axial dimensions reported for each diffraction pattern are defined as the approximate width and length of each of the traps within the equipotential energy surface equal to the trap depth. Tables I and II quantitatively illustrate the effects of changing either the distance between the lens and the diffracting aperture or the size of the diffracting aperture on the depth, dimensions, and axial-radial aspect ratio of the trap sites.

TABLE II. Properties of the primary BDT for various diffraction patterns. For all patterns, the lens focal length is $40 \mathrm{~mm}$, the detuning parameter is set to $=10^{3} \Gamma$, and the trap depth is given per $100 \mathrm{~W} / \mathrm{m}^{2}$ of light intensity incident upon the aperture.

\begin{tabular}{|c|c|c|c|c|}
\hline Diffraction pattern & $\begin{array}{l}\text { Figure } \\
\text { number }\end{array}$ & $\begin{array}{l}\text { BDT trap depth } \\
\text { per } 100 \mathrm{~W} / \mathrm{cm}^{2} \\
(\mu \mathrm{K})\end{array}$ & $\begin{array}{l}\text { Radial width } \\
\qquad(\mu \mathrm{m})\end{array}$ & $\begin{array}{l}\text { Axial length } \\
\quad(\mu \mathrm{m})\end{array}$ \\
\hline $\begin{array}{l}\text { Original } \\
a=25 \mu \mathrm{m}\end{array}$ & 3(a) & 820 & 9.05 & 125 \\
\hline $\begin{array}{l}\text { Projected } \\
a=25 \mu \mathrm{m}, L=80.4 \mathrm{~mm} \\
\text { Projected }\end{array}$ & $3(b)$ & 823 & 9.05 & 125 \\
\hline $\begin{array}{l}a=25 \mu \mathrm{m}, L=125 \mathrm{~mm} \\
\text { Projected }\end{array}$ & $3(\mathrm{c}), 3(\mathrm{e})$ & 3680 & 4.26 & 27.9 \\
\hline $\begin{array}{l}a=25 \mu \mathrm{m}, L=68 \mathrm{~mm} \\
\text { Original }\end{array}$ & $3(d)$ & 393 & 13.1 & 264 \\
\hline $\begin{array}{l}a=5 \mu \mathrm{m} \\
\text { Projected nanotrap }\end{array}$ & 4(a) & 818 & 1.86 & 5.24 \\
\hline$a=5 \mu \mathrm{m}, L=125 \mathrm{~mm}$ & 4(b) & 3700 & 0.85 & 1.11 \\
\hline
\end{tabular}




\section{CONCLUSION}

Scalar diffraction theory and beam-propagation methods have been employed to overcome two experimental challenges with using diffraction patterns to optically trap atoms: the requirement for the diffracting plane or mask to be placed inside the vacuum chamber and the inability to adjust the trap properties for a given diffracting aperture. The model presented here uses a single lens to project the diffraction pattern found just beyond a circular aperture to an optics-free region beyond the lens. An integral method has been used to calculate the complex scalar field of the diffracted light for any point of interest in the region beyond the projecting lens. For quickly determining the location and spacing of the projected maxima and minima locations, an analytical model can be used for on-axis locations. Calculations show that control of the trap depth and size is possible by adjustment of the aperture to lens distance and that additional control over the axial-radial aspect ratio is possible by changing the size of the diffracting aperture. Although the model presented here is for a single circular aperture producing a single projected atom trap, the model can be extended to one-dimensional or two-dimensional arrays of traps using a diffracting mask of one-dimensional or two-dimensional arrays of pinholes.

\section{ACKNOWLEDGMENTS}

K.G.-C. acknowledges support by the National Science Foundation (Grant No. PHY-0855524).
[1] J. E. Bjorkholm, R. R. Freeman, A. Ashkin, and D. B. Pearson, Phys. Rev. Lett. 41, 1361 (1978).

[2] R. Grimm, M. Weidemüller, and Y. B. Ovchinnikov, Adv. At. Mol. Opt. Phys. 42, 95 (2000).

[3] N. Friedman, A. Kaplan, and N. Davidson, Adv. At. Mol. Opt. Phys. 48, 99 (2002).

[4] S. Chu, J. E. Bjorkholm, A. Ashkin, and A. Cable, Phys. Rev. Lett. 57, 314 (1986).

[5] P. Verkerk, B. Lounis, C. Salomon, C. Cohen-Tannoudji, J.-Y. Courtois, and G. Grynberg, Phys. Rev. Lett. 68, 3861 (1992).

[6] A. Hemmerich and T. W. Hänsch, Phys. Rev. Lett. 70, 410 (1993).

[7] G. Grynberg, B. Lounis, P. Verkerk, J.-Y. Courtois, and C. Salomon, Phys. Rev. Lett. 70, 2249 (1993).

[8] Y. B. Ovchinnikov, I. Manek, A. I. Sidorov, G. Wasik, and R. Grimm, Europhys. Lett. 43, 510 (1998).

[9] T. Kuga, Y. Torii, N. Shiokawa, T. Hirano, Y. Shimizu, and H. Sasada, Phys. Rev. Lett. 78, 4713 (1997).

[10] M. Hammes, D. Rychtarik, B. Engeser, H.-C. Nägerl, and R. Grimm, Phys. Rev. Lett. 90, 173001 (2003).
[11] K. Christandl, G. P. Lafyatis, S.-C. Lee, and J.-F. Lee, Phys. Rev. A 70, 032302 (2004).

[12] V. V. Klimov and V. S. Letokhov, Opt. Commun. 121, 130 (1995).

[13] G. D. Gillen, S. Guha, and K. Christandl, Phys. Rev. A 73, 013409 (2006).

[14] T. N. Bandi, V. G. Minogin, and S. N. Chormaic, Phys. Rev. A 78, 013410 (2008).

[15] L. Chen and J. Yin, Phys. Rev. A 80, 065401 (2009).

[16] G. Bekefi, J. Appl. Phys. 24, 1123 (1953).

[17] S. Guha and G. D. Gillen, Opt. Express 13, 1424 (2005).

[18] G. D. Gillen and S. Guha, Am. J. Phys. 72, 1195 (2004).

[19] J. W. Goodman, Introduction to Fourier Optics, 3rd ed. (Roberts, Englewood, CO, 2005).

[20] A. H. Barnett, S. P. Smith, M. Olshanii, K. S. Johnson, A. W. Adams, and M. Prentiss, Phys. Rev. A 61, 023608 (2000).

[21] L. J. Curtis, Atomic Structure and Lifetimes: A Conceptual Approach (Cambridge University Press, Cambridge, 2003), Chap. 6.

[22] H. J. Metcalf and P. van der Straten, Laser Cooling and Trapping (Springer, New York, 1999). 